\title{
Reprocessing the Hipparcos data of evolved stars
}

\section{Revised Hipparcos period-luminosity relationship for galactic long-period variable stars ${ }^{\star, \star \star}$}

\author{
G. R. Knapp ${ }^{1}$, D. Pourbaix ${ }^{1,2, \star \star \star}$, I. Platais ${ }^{3}$, and A. Jorissen ${ }^{2, \star \star \star}$ \\ 1 Department of Astrophysical Sciences, Princeton University, Princeton, NJ 08544, USA \\ 2 Institut d'Astronomie et d'Astrophysique, Université Libre de Bruxelles, CP 226, Boulevard du Triomphe, 1050 Bruxelles, \\ Belgium \\ e-mail: pourbaix; ajorisse@astro.ulb.ac.be \\ 3 Department of Physics and Astronomy, The Johns Hopkins University, 3400 North Charles Street, Baltimore, MD 21218, \\ USA \\ e-mail: imants@pha.jhu.edu
}

Received 29 January 2003 / Accepted 21 March 2003

\begin{abstract}
We analyze the $K$ band luminosities of a sample of galactic long-period variables using parallaxes measured by the Hipparcos mission. The parallaxes are in most cases re-computed from the Hipparcos Intermediate Astrometric Data using improved astrometric fits and chromaticity corrections. The $K$ band magnitudes are taken from the literature and from measurements by COBE, and are corrected for interstellar and circumstellar extinction.

The sample contains stars of several spectral types: M, S and C, and of several variability classes: Mira, semiregular SRa, and $\mathrm{SRb}$. We find that the distribution of stars in the period-luminosity plane is independent of circumstellar chemistry, but that the different variability types have different $\mathrm{P}-\mathrm{L}$ distributions. Both the Mira variables and the SRb variables have reasonably well-defined period-luminosity relationships, but with very different slopes. The SRa variables are distributed between the two classes, suggesting that they are a mixture of Miras and SRb, rather than a separate class of stars. New period-luminosity relationships are derived based on our revised Hipparcos parallaxes. The Miras show a similar period-luminosity relationship to that found for Large Magellanic Cloud Miras by Feast et al. (1989).

The maximum absolute $K$ magnitude of the sample is about -8.2 for both Miras and semi-regular stars, only slightly fainter than the expected AGB limit. We show that the stars with the longest periods $(P>400 \mathrm{~d})$ have high mass loss rates and are almost all Mira variables.
\end{abstract}

Key words. stars: carbon - stars: AGB and post-AGB - stars: oscillations

\section{Introduction}

This paper investigates the period-luminosity $(\mathrm{P}-\mathrm{L})$ relationship, or relationships, for long-period variable stars (LPV) in the solar neighborhood. P-L relationships for stars in the instability strips of the HR diagram have proven to be of fundamental value in using variable stars as secondary distance indicators. Stellar variability is also a powerful tool for studying stellar structure, chemistry and mass loss (e.g. Wood 1990; Willson 2000; Alvarez et al. 2001). The present paper

Send offprint requests to: G. R. Knapp,

e-mail: gk@astro.princeton.edu

* Based on observations from the Hipparcos astrometric satellite operated by the European Space Agency (ESA 1997).

$\star \star$ Table 1 is only available in electronic form at the CDS via anonymous ftp to cdsarc.u-strasbg.fr $(130.79 .128 .5)$ or via http://cdsweb.u-strasbg.fr/cgi-bin/qcat?J/A+A/403/993

$\star \star \star$ Research Associate, FNRS, Belgium. investigates whether one or more $\mathrm{P}-\mathrm{L}$ relationships exist for LPVs in the local region of the Galaxy, using re-derived Hipparcos parallaxes, $K$ magnitude data from the literature, and $K$-band flux densities and variability data derived from the COBE DIRBE time-series data (Boggess et al. 1992; Hauser et al. 1998).

The P-L relationship for LPVs has been investigated using the Hipparcos Catalogue (van Leeuwen et al. 1997; Whitelock \& Feast 2000) and for samples of objects at the same distance - in the Large Magellanic Cloud (Glass \& Lloyd Evans 1981; Feast et al. 1989; Groenewegen \& Whitelock 1996; Cioni et al. 2001), and in the bulge of the Galaxy (Alard et al. 2001). The last two papers make use of the vast data compilation on variable stars available from microlensing surveys. These papers find P-L relationships for LMC Miras with both oxygen and carbon chemistry, and relationships for semi-regular (SR) variables which have the same slope as for Miras but with two sequences suggested to correspond to the long and short 
periods which often coexist in these stars (Cioni et al. 2001; see also the discussion by Szatmary et al. 1996; Bedding et al. 1998; Zijlstra et al. 2002). In the galactic bulge, Alard et al. (2001) find a P-L relationship for Mira variables but no relation for SR variables. Given the potential use of these stars as secondary distance indicators (their luminosities are high, $\geq 3000 L_{\odot}$ and their effective temperatures low, $\leq 2500 \mathrm{~K}$, so that the bulk of their flux is emitted at near-infrared wavelengths, where interstellar extinction is much lower than at optical wavelengths), the establishment of a well-defined P$\mathrm{L}$ relationship is of a significant interest. Further, the $\mathrm{P}-\mathrm{L}$ relationship establishes the mode in which the star is pulsating, and gives information on the effect of pulsation on mass loss.

Measurements of the P-L relationship in the LMC and the galactic bulge provide the slope $a$ of the relationship:

$M_{K}=a \log P($ days $)+b$

and the zero-point $b$ (using the known distances to the LMC and the bulge). However, models (e.g. Wood 1990, 2000) show that the zero-point absolute luminosity is metallicity dependent. Comparisons of the $\mathrm{P}-\mathrm{L}$ relationship derived for nearby galactic LPVs, whose absolute distances are known from parallax measurements and for which there is much more information available on metallicity and mass loss, are thus worth investigating.

Establishment of P-L relationships for nearby LPVs is difficult, however. Even after Hipparcos (ESA 1997) there are relatively few well determined parallaxes for these rare, luminous stars, and their use requires attention to statistical biases (cf. Arenou \& Luri 1999). Several studies have assumed that the $\mathrm{P}-\mathrm{L}$ relationship for nearby Miras has the same slope as found for the LMC Miras (Groenewegen \& Whitelock 1996; Bedding \& Zijlstra 1998; Whitelock \& Feast 2000; Bergeat et al. 2001) and have used this relationship to calculate the zero point for galactic populations. Barthès et al. (1999), however, using both direct and statistical parallaxes for a sample of Miras, find a different slope than that seen in the LMC, while Alvarez et al. (1997) identify two groups of Miras with different zero points (cf. also Jura \& Kleinmann 1992).

Hipparcos provided the first parallaxes for most LPVs for which this information is now available. However, most of the parallaxes available even from Hipparcos are measured at low confidence. Added to this are reduction problems associated with the very red colors and extreme variability of these objects. These factors make the evaluation of absolute magnitudes for these objects quite uncertain.

We have recently undertaken a re-analysis of the Hipparcos astrometric data for red giant stars with the aim of improving the accuracy of the parallax information for these objects. The re-reduction scheme is described in detail by Platais et al. (2003) and Pourbaix et al. (2003). The present paper uses improved parallax information from these studies, plus some Hipparcos catalogue parallaxes, to re-examine the absolute magnitudes of variable AGB stars. We work entirely in $K$ magnitudes, using magnitude data from the literature and from the COBE-DIRBE data. We also incorporate corrections for interstellar and circumstellar extinction, and use the DIRBE
Table 1. Stars for which a new solution based on the Hipparcos observations was derived. $\varpi$ and $\varepsilon(\varpi)$ are the parallax and its error in mas. $\Delta \varpi \equiv \frac{\varpi-\varpi_{\mathrm{HIP}}}{\sigma_{\varpi_{\mathrm{HP}}}}$ is the difference between the revised parallax and the published one reckoned in units of the standard error in the Hipparcos parallax, $A_{K}$ is the total interstellar and circumstellar extinction in the $K$ band (see text). $K$ is the $2.2 \mu$ m apparent $K$ magnitude. $P$ is the period in days, "var" is the variability type from CGCVS, "Chem" is the carbon/oxygen chemistry of the atmosphere.

\begin{tabular}{llllllllll}
\hline \hline HIP & CGCVS & $\varpi$ & $\varepsilon(\varpi)$ & $\Delta \varpi$ & $A_{K}$ & $K$ & $P$ & var & Chem \\
\hline
\end{tabular}

data to examine the $K$ band variability of the stars. The assembly of the data set is described in the next Section. Section 3 discusses the distribution of LPVs in the P-L plane and investigates their $\mathrm{P}-\mathrm{L}$ relationships. We also examine the maximum AGB luminosity for both carbon- and oxygen-rich stars. Section 4 briefly discusses data on period variability, from the literature and from the DIRBE data, and discusses the relationships among the different types of LPVs. Section 5 discusses Mira variables with long periods and large mass loss rates.

\section{Data}

The sample of objects discussed herein was basically defined by the available data: parallaxes, $K$ magnitudes, periods and variability types. Secondary information includes the position (which together with the parallax allows the calculation of interstellar extinction); the $12 \mu \mathrm{m}$ flux density (Beichman et al. 1988) which, together with the $2 \mu \mathrm{m}$ flux density, gives an estimate for the circumstellar extinction, and the circumstellar chemistry, since the dust opacity at $12 \mu \mathrm{m}$ is strongly dependent on its composition.

The data are given in Tables 1 and 2, where we list: the Hipparcos catalogue number; the variable star name; the parallax $\varpi$ in milliarcseconds (mas); the parallax error $\varepsilon(\varpi)$ in mas; the total (interstellar and circumstellar) $K$ band extinction $A_{K}$; the $K$ magnitude; the period in days; the variability type; and the chemistry $(\mathrm{O}=$ "oxygen-rich", i.e. $n(\mathrm{O})>n(\mathrm{C}) ; \mathrm{C}=$ carbon star and $\mathrm{S}=$ zirconium-enhanced star). The sources of these various quantities are discussed below.

\subsection{Parallaxes}

The sample of LPVs analyzed in this work was selected from stars observed by Hipparcos (ESA 1997). This mission provided accurate astrometric measurements for a sample of some 120000 stars which is approximately complete to a brightness limit of $V \sim 8$ but also contains many fainter stars. Included in the Hipparcos input catalogue were several hundred evolved cool giant stars, and Hipparcos provided the first measured parallaxes for almost all of these. However, because of their relatively large distances, variability, red colors and, for some stars, their faint apparent magnitudes, the parallax errors for these stars are often larger than the nominal Hipparcos $1 \sigma$ accuracy of 1 mas. We have therefore undertaken a re-analysis of the Hipparcos Intermediate Astrometric Data (IAD) for these 
Table 2. Same as Table 1 but for $\mathrm{SRb}$ variables for which the Hipparcos data have not been reprocessed.

\begin{tabular}{llllllll}
\hline \hline HIP & CGCVS & $\varpi$ & $\varepsilon(\varpi)$ & $A_{K}$ & $K$ & $P$ & Chem. \\
\hline 13064 & Z Eri & 4.07 & 0.90 & 0.01 & 0.32 & 80 & $\mathrm{O}$ \\
13384 & RR Eri & 2.96 & 1.04 & 0.01 & 0.52 & 97 & $\mathrm{O}$ \\
19116 & CY Eri & 2.87 & 1.00 & 0.01 & 1.96 & 25 & $\mathrm{O}$ \\
21046 & RV Cam & 3.01 & 0.97 & 0.06 & 0.52 & 101 & $\mathrm{O}$ \\
23680 & W Ori & 4.66 & 1.44 & 0.07 & -0.25 & 212 & $\mathrm{C}$ \\
24169 & RX Lep & 7.30 & 0.71 & 0.01 & -1.40 & 60 & $\mathrm{O}$ \\
28558 & DP Ori & 5.34 & 1.73 & 0.01 & 1.05 & 90 & $\mathrm{O}$ \\
28874 & S Lep & 3.63 & 0.78 & 0.02 & -0.46 & 89 & $\mathrm{O}$ \\
34922 & LZ Pup & 16.46 & 1.27 & 0.02 & -2.49 & 140.6 & $\mathrm{O}$ \\
38406 & BC CMi & 7.77 & 0.99 & 0.00 & 0.83 & 35 & $\mathrm{O}$ \\
41664 & RT Hya & 3.68 & 1.02 & 0.01 & 0.09 & 290 & $\mathrm{O}$ \\
42502 & AK Hya & 5.12 & 0.92 & 0.02 & -0.61 & 75 & $\mathrm{O}$ \\
44050 & RT Cnc & 2.94 & 1.11 & 0.01 & 0.20 & 60 & $\mathrm{O}$ \\
55639 & T Crt & 5.04 & 1.06 & 0.01 & 1.20 & 70 & $\mathrm{O}$ \\
61022 & BK Vir & 5.68 & 1.12 & 0.01 & -0.88 & 150 & $\mathrm{O}$ \\
62611 & SV Crv & 2.72 & 0.97 & 0.01 & 1.07 & 70 & $\mathrm{O}$ \\
63642 & RT Vir & 7.25 & 1.10 & 0.01 & -1.12 & 155 & $\mathrm{O}$ \\
64569 & SW Vir & 7.00 & 1.20 & 0.01 & -1.87 & 150 & $\mathrm{O}$ \\
66666 & V744 Cen & 6.00 & 0.76 & 0.02 & -0.72 & 90 & $\mathrm{O}$ \\
71644 & RV Boo & 2.54 & 0.98 & 0.00 & -0.01 & 137 & $\mathrm{O}$ \\
73213 & FY Lib & 2.97 & 1.19 & 0.03 & 0.12 & 120 & $\mathrm{O}$ \\
78574 & X Her & 7.26 & 0.70 & 0.01 & 0.67 & 95 & $\mathrm{O}$ \\
81188 & TX Dra & 3.52 & 0.56 & 0.01 & 1.38 & 78 & $\mathrm{O}$ \\
82249 & AH Dra & 3.39 & 0.70 & 0.01 & 0.57 & 158 & $\mathrm{O}$ \\
87850 & OP Her & 3.26 & 0.54 & 0.02 & 0.05 & 120.5 & $\mathrm{O}$ \\
89669 & IQ Her & 3.82 & 0.87 & 0.04 & 0.04 & 75 & $\mathrm{O}$ \\
91781 & V3879 Sgr & 2.67 & 1.01 & 0.05 & -0.42 & 50 & $\mathrm{O}$ \\
92862 & R Lyr & 9.33 & 0.52 & 0.01 & -2.10 & 46 & $\mathrm{O}$ \\
96204 & V450 Aq1 & 3.80 & 0.90 & 0.04 & -0.08 & 64.2 & $\mathrm{O}$ \\
97151 & V973 Cyg & 3.60 & 0.57 & 0.02 & 1.49 & 40 & $\mathrm{O}$ \\
100935 & T Mic & 3.44 & 1.19 & 0.01 & -1.59 & 347 & $\mathrm{O}$ \\
106642 & W Cyg & 5.28 & 0.63 & 0.02 & -1.48 & 131.1 & $\mathrm{O}$ \\
1107487 & AG Cap & 2.64 & 0.85 & 0.01 & 1.20 & 25 & $\mathrm{O}$ \\
\hline & UW Peg & 3.38 & 1.57 & 0.01 & 2.27 & 106 & $\mathrm{O}$ \\
\hline & S Phe & 3.93 & 0.87 & 0.03 & 1.56 & 141 & $\mathrm{O}$ \\
\hline & & & & & &
\end{tabular}

objects. The re-processing scheme contains two components: deriving epoch $V-I$ photometry for each IAD using the method described by Platais et al. (2003), and then reprocessing the IAD to derive parallaxes using epoch chromaticity corrections. This accounts for the color dependence of the Hipparcos detector, a particularly important aspect of the data processing for the very red, and highly variable, LPVs. For the present study, we used three data sets based on these new reductions: carbon stars - the requirement of a measured period removes the much less luminous R type stars from the sample (cf. Knapp et al. 2001), leaving the cooler, more luminous, variable N-type carbon stars; Variability-Induced Movers (VIMs; Pourbaix et al. 2003); and oxygen-rich Miras. The parallaxes and their errors are given in Table 1 . Note that in many cases the parallax is not significant.

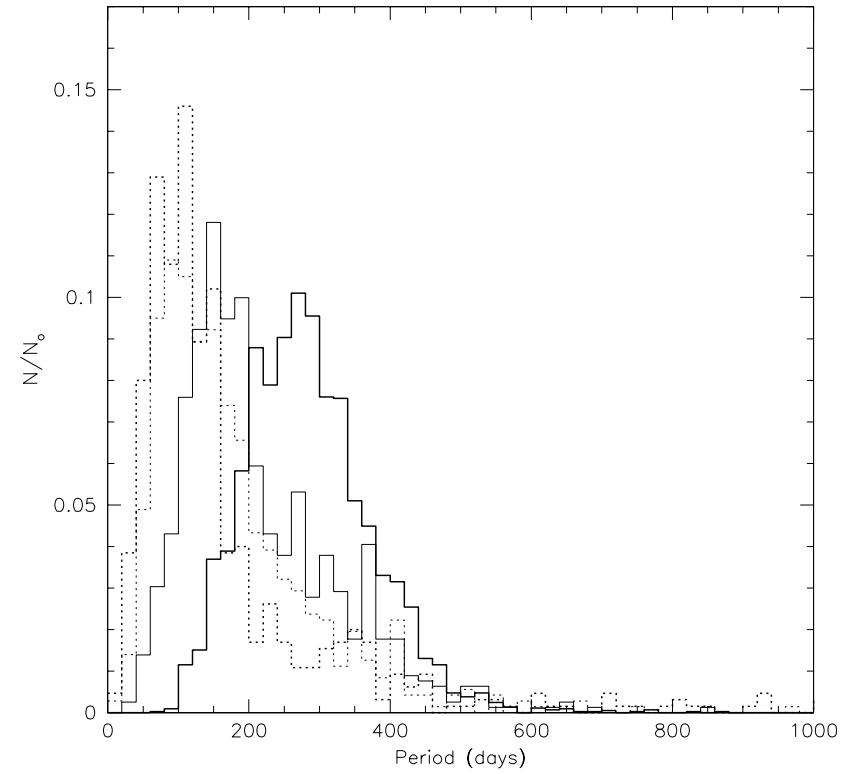

Fig. 1. Frequency of periods from the CGCVS. Heavy solid line: Mira. Light solid line: SRa. Heavy dotted line: SRb. Light dotted line: SR.

The samples of carbon stars, VIMs and oxygen-rich Miras defined above are complete in the sense that they comprise all such objects observed by Hipparcos except for the small number without measured periods or $K$ magnitudes. The Hipparcos carbon star and VIM samples contribute a fair number of SR variables: to round out the data sets, we extracted from the Hipparcos catalogue data for the remaining oxygen-rich SRb observed by Hipparcos. The data for this sample, with the same information as in Table 1, are given in Table 2. Two stars with the DMSA/X solution, namely HIP 40977 and 87820, were ignored in this investigation because of the low reliability of their IAD. However, HIP 91389, also a DMSA/X entry, was included because it is successfully processed with the VIM model (Pourbaix et al. 2003).

\subsection{Periods and variability types}

The monumental combined general catalogue of variable stars (CGCVS, Kholopov et al. 1998) contains data for many types of variable stars, including amplitudes at visual wavelengths, periods, and classification. LPVs are classified into several subtypes: Miras, with long, well-established, repeatable periods (typically $300 \mathrm{~d}$ or longer) and large amplitudes $(\Delta V>$ $2.5 \mathrm{mag}$ ): semiregular variables with shorter, less repeatable periods and smaller amplitudes, which are subclassified into types SRa and SRb: and irregular variables, type Lb, which show no well-defined period (in fact, periods have been measured for a few of these, and their variability behavior is indistinguishable from that of SRb; Kerschbaum et al. 2001). Many semiregular variables show two or more periods, and some switch between two or more periods. We discuss these variability types further in Sect. 5 .

Figure 1 shows the period distribution for LPVs from the CGCVS. Many of the semiregular variables are classified only as SR. The period distributions for all types cover a similar 
range, and there are few stars of any type with periods longer than 400 days (we discuss this further in Sect. 5). The median period of the Miras is more than twice that of the SRb. The stars classified only as SR have a period distribution closest to the $\mathrm{SRb}$. The SRa distribution is intermediate between the Miras and SRb. In this paper, we will use three classes: Mira, SRa and SRb, combining the last with the SR and the very small number of stars classified as $\mathrm{Lb}$ for which a period has been found.

The periods and variability types in Table 1 were taken from the CGCVS and the Hipparcos variability annex. Periods measured by Hipparcos agree well with the CGCVS periods in almost all cases.

\section{3. $K$ magnitudes}

We analyzed the P-L relation for LPVs using $K$ magnitudes, which are available for almost all bright LPVs from the literature. The variation of Miras between maximum and minimum light can exceed $10 \mathrm{mag}$ at visual wavelengths, since the visual luminosity is a highly non-linear function of photospheric temperature because of molecule formation (for a recent discussion see Reid \& Goldston 2002); while the variability amplitude is much smaller (about $0.5 \mathrm{mag}$ ) at $K$ (e.g. Whitelock et al. 2000). The $K$-band luminosity is also less affected by chemical composition (Wood 2000) and is near the peak of the stellar spectral energy distribution.

$K$ magnitudes were taken from the literature, including the IRC (Neugebauer \& Leighton 1969), the compilation by Gezari et al. (1999), Kerschbaum \& Hron (1994) and Whitelock et al. (2000). Except for the last reference, which gives $K$ magnitudes averaged over the light curve, these are single-epoch observations.

To study the variance of the $K$ magnitudes caused by variability, we extracted $2.2 \mu \mathrm{m}$ light curves from the data from the diffuse infrared background explorer (DIRBE Hauser et al. 1998) on the cosmic background explorer COBE (Boggess et al. 1992). DIRBE provides well-sampled partial light curves (sometimes with several observations per day) for bright point sources at wavelengths from $1.25 \mu \mathrm{m}$ to $240 \mu \mathrm{m}$ over a time interval of almost 300 days (11 December 1989 to 21 September 1990). The gaps in the data and the short duration of the COBE mission relative to the typical period of an LPV limit the usefulness of COBE for measuring periods (see e.g. Smith et al. 2002). Because of the large DIRBE beam $\left(40^{\prime}\right)$ the data are useful only for the brightest point sources, those brighter than about $0-1 \mathrm{mag}$.

The DIRBE $2.2 \mu \mathrm{m}$ light curves were extracted for the brightest objects in Table 1 (approximately 0 mag and brighter at $K$ ) in the present sample using software written by $\mathrm{N}$. Odegard and D. Leisawitz and available at http://cobe.gsfc.nasa.gov/cio/browser.html. The light curves of some of these stars will be discussed in Sect. 4 . Figure 2 shows the DIRBE $K$ magnitudes versus those from the literature. The DIRBE $2.2 \mu \mathrm{m}$ magnitudes were calculated from the observed flux densities assuming a value for the flux

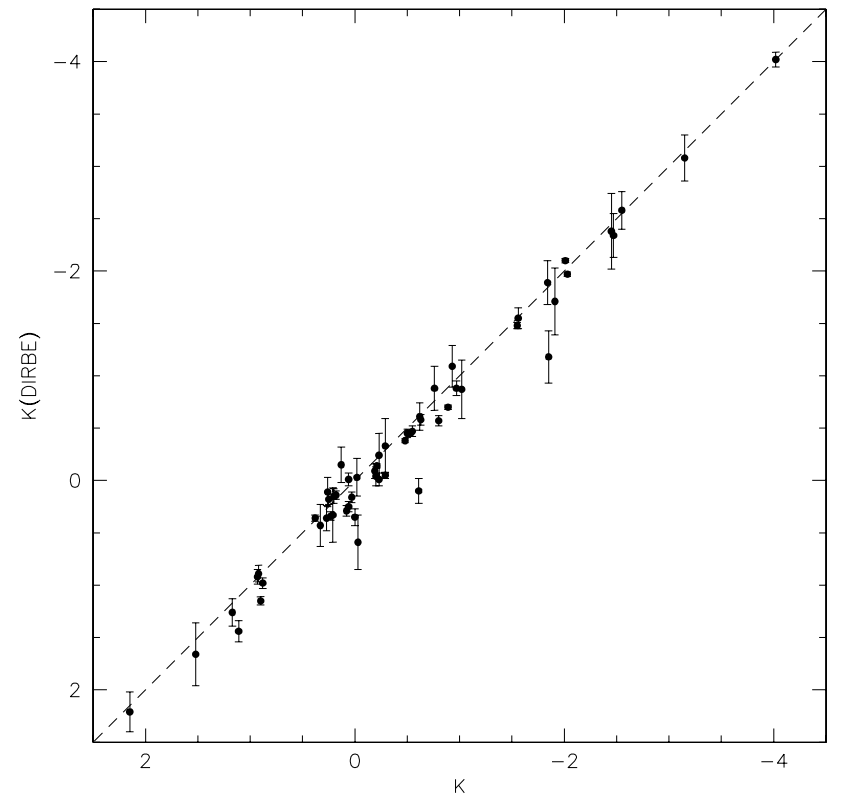

Fig. 2. $K$ magnitude from DIRBE $2.2 \mu \mathrm{m}$ flux densities vs. $K$ magnitude from the literature. Equal magnitudes are indicated by the dashed line. The bars on the DIRBE magnitudes show the observed range of magnitude variation over the time span of the COBE observations.

density of a 0 mag star of $654 \mathrm{Jy}$ (using the calibrations of a 0.03 mag star by Bessell \& Brett 1988).

The DIRBE time series data were used to calculate an average flux density for the entire data set plus the mean deviation from that average. In most cases most of the deviation is found to be due to stellar variability rather than noise. The ranges in magnitudes for the COBE data are also shown in Fig. 2.

In general the agreement between the data from the literature and from DIRBE is excellent. The mean internal deviation from the COBE data is 0.2-0.4 magnitudes for Mira variables and about $0.02-0.1 \mathrm{mag}$ for SR and SRb variables. In almost all cases the difference between the DIRBE and the literature magnitude is within these deviations, which therefore dominate the measurement noise. The data for a few stars show larger discrepancies, and may be due to longer-term variations in these stars.

Figure 3 shows the ratio of the maximum to minimum $2 \mu \mathrm{m}$ flux density for stars for which a sufficient portion of the light curve is observed by DIRBE to observe at least one maximum and one minimum (only a minority of the stars in Table 1). Figure 3a shows this ratio versus the time difference between the minimum and maximum, or vice-versa - not necessarily half the period, since the light curves for LPVs are often asymmetric. Figure $3 \mathrm{~b}$ shows the ratio versus the period from the CGCVS. Figure 3 shows that Miras are far more readily distinguished from $\mathrm{SRb}$ by amplitude than by period (cf. also Cioni et al. 2001), although stars with $\left(t_{\max }-t_{\min }\right)$ shorter than about 70 days are always SRb. The SRa variables overlap both the Miras and SRb and are therefore likely to be a mixture of the two types (cf. Fig. 1).

In the next section, then, we discuss only two samples: Miras and SR/SRb. We do not include the SRa in the analysis. We assume that the error in the COBE median $K$ magnitude 

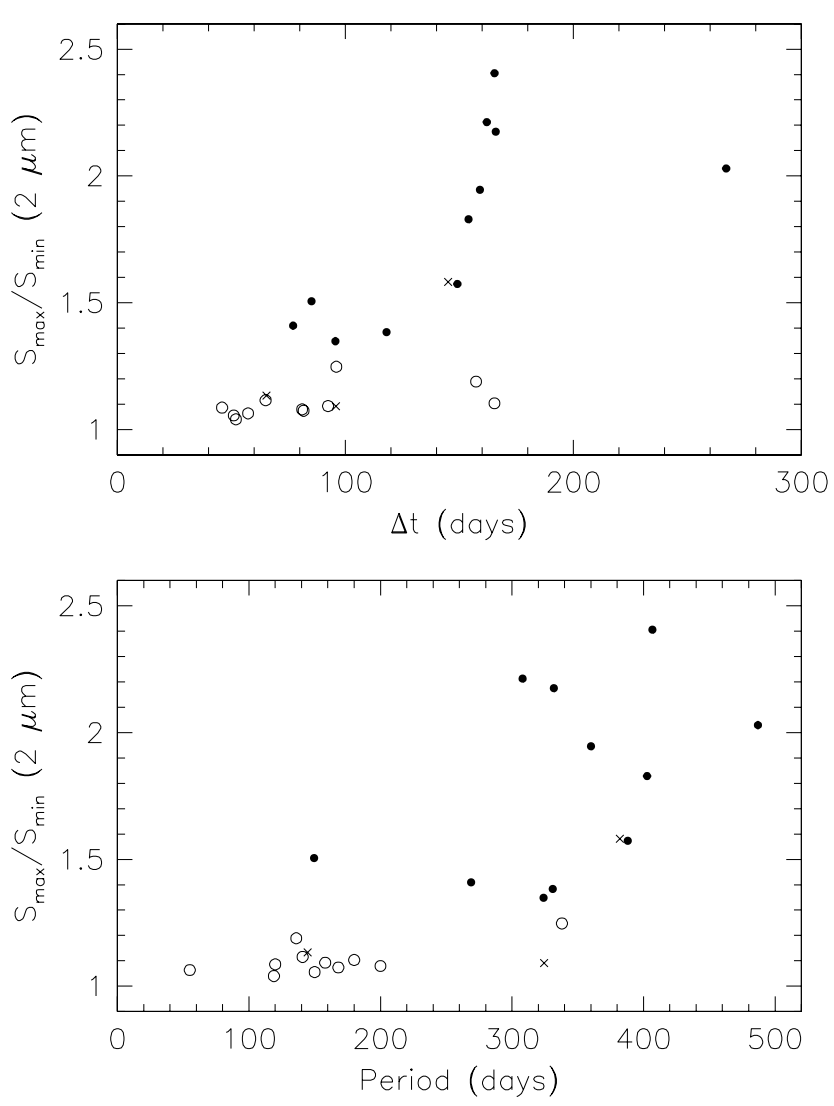

Fig. 3. Ratio of maximum and minimum $2.2 \mu \mathrm{m}$ flux densities versus (a) time between maximum and minimum $2.2 \mu \mathrm{m}$ light, as measured by DIRBE (upper panel) and (b) period from the CGCVS (lower panel). Filled circles: Mira variables. Open circles: SRb and SR variables. Crosses: SRa variables.

is $0.1 \mathrm{mag}$ for SR and $0.3 \mathrm{mag}$ for Miras, in both cases dominated by variability; these are the median values found from the COBE light curves.

\subsection{Extinction}

The extinction towards the stars has two components, interstellar and circumstellar. We used the model of Hakkila et al. (1997) to calculate $A_{V}$, the total interstellar extinction in $V$, from the position of each star and its distance, estimated from $\varpi^{-1}$. The $K$ band interstellar extinction is then found from $A_{K_{i}}=0.114 A_{V}$ (Cardelli et al. 1989).

Many of the stars in Table 1 are losing mass and are surrounded by dusty circumstellar envelopes. Ivezić \& Elitzur (1997) show that the resulting spectral energy distribution is a function of the dust composition and the optical depth. The circumstellar extinctions for the stars discussed in this paper are not large by the standards of mass-losing AGB stars (they are all visible objects) and the optical depth can be estimated from the ratio of the $12 \mu \mathrm{m}$ flux density (emitted almost entirely by the dusty envelope) to the $2 \mu \mathrm{m}$ flux density (mostly emitted by the photosphere). The relationship between the $12 \mu \mathrm{m} / 2 \mu \mathrm{m}$ flux ratio and the $K$ band circumstellar extinction $A_{K_{c}}$ was estimated using a spherically symmetric dusty envelope model (Knapp et al. 1993) using silicate grains for oxygen and S stars (cf. Jorissen \& Knapp 1998) and carbonaceous grains for carbon stars. The model assumed condensation temperatures of $800 \mathrm{~K}$ and $1000 \mathrm{~K}$ for silicate and carbonaceous grains respectively (cf. Ivezić \& Elitzur 1997) to define the radius of the inner edge of the model envelope. The model's central star is a blackbody with $T_{\star}=2500 \mathrm{~K}, L_{\star}=5000 L_{\odot}$, for which the intrinsic ratio is $S_{12} / S_{2.2}=0.18$. For low optical depths

$A_{K_{c}} \approx 0.07\left(\frac{S_{12}}{S_{2.2}}-0.18\right)$

for silicate envelopes, and

$A_{K_{c}} \approx 1.1\left(\frac{S_{12}}{S_{2.2}}-0.18\right)$

for carbonaceous dust. The large difference between the two is because of the strong contribution of the silicate $9.7 \mu \mathrm{m}$ emission feature in the IRAS $12 \mu \mathrm{m}$ band. A very small number of stars have no IRAS observations: we assume a circumstellar extinction of 0 mag for these stars.

The total (interstellar + circumstellar) extinction in the $K$ band is listed in Tables 1 and 2 . The uncertainties in both the interstellar and circumstellar extinction are large, but the contribution of these errors to the errors in the computed absolute $K$ magnitude are dwarfed by those in the parallax and by stellar variability, and thus will be neglected in the remainder of this paper.

\section{The period-luminosity relationship}

The stars listed in Tables 1 and 2 often have poorly measured parallaxes. Figure 4 shows the distribution in the period- $M_{K}$ plane of stars with reliable $\varpi$, defined by:

- probability that $\varpi>0$ at the $95 \%$ confidence level. The mean Hipparcos parallax error is $\sim 1$ mas with some small scatter around this value depending on the location of the star on the sky and on its brightness. LPVs are very luminous, so many observed by Hipparcos may be at distances $>1 \mathrm{kpc}$. The probability that the parallax is detected is determined by including/not including the parallax in the astrometric solution, and defining the probability that the parallax is detected using the F-test (cf. Pourbaix et al. 2003);

- $\varpi / \varepsilon(\varpi)>2$;

$-\varpi>0$;

$-\varepsilon(\varpi)<2$ mas. As discussed by Platais et al. (2003) the errors on the Hipparcos parallaxes for some relatively bright red giant stars are anomalously large, and measurements with large errors often produce inaccurate parallaxes.

The distance is calculated from $D=1000 / \varpi$ and the absolute magnitude $M_{K}$ from this distance and the observed $K$ magnitude, corrected for circumstellar and interstellar extinction. The errors on $M_{K}$ are assumed to be due to the errors (or rather range of photometric variation) in $K$ and the parallax error. In Fig. 4, the stars are coded by variability type (Mira, SR/SRb and SRa) and by circumstellar chemistry (combining the oxygen and S stars). Also shown in Fig. 4 is the period-luminosity 

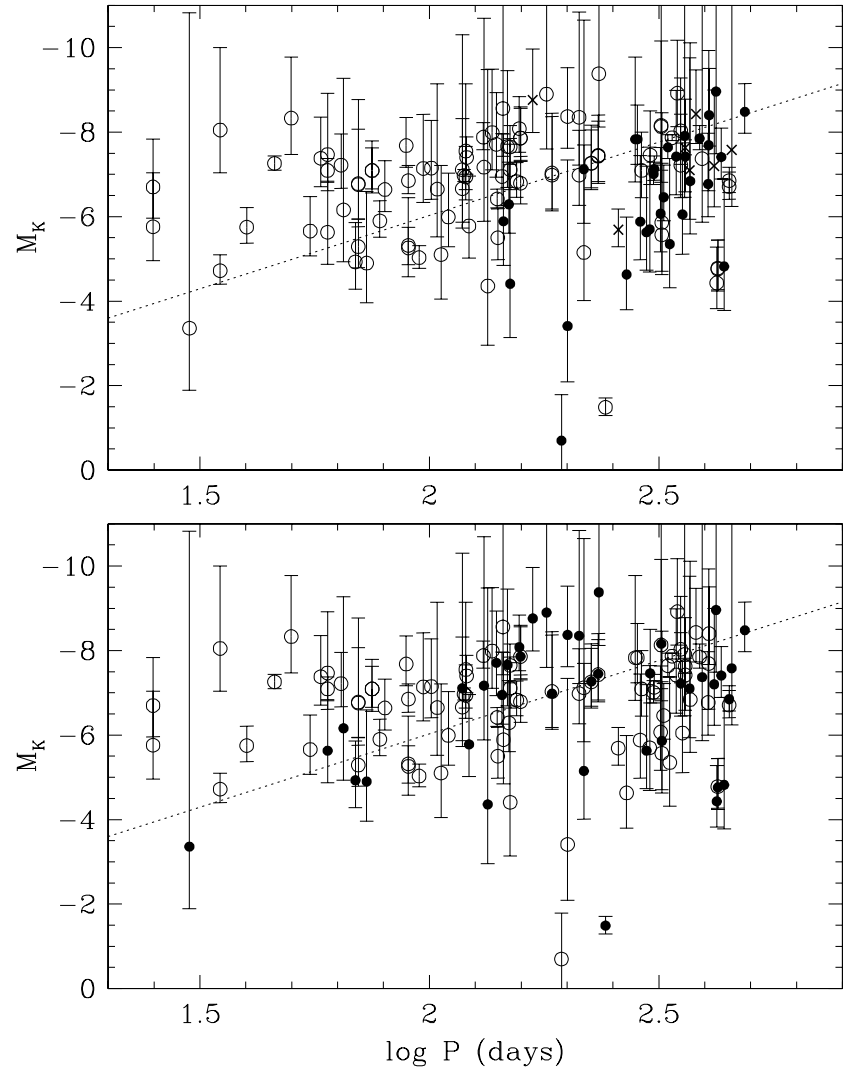

Fig. 4. Distribution of LPVs in the $M_{K}-\log P$ plane. (a) Upper panel: stars are sorted by variable type. Filled circles: Miras. Open circles: SR/SRb. Crosses: SRa. (b) Lower panel: stars are sorted by chemical type. Filled symbols: carbon stars. Open symbols: oxygen stars. The dashed line is the P-L relationship of Eq. (4).

relationship for LMC Miras by Feast et al. (1989) with the zero point as determined for galactic Miras by Whitelock \& Feast (2000):

$M_{K}=-3.47 \log P($ days $)+0.85$.

Figure 4 shows several features:

1. To first order, the location of LPVs on the P-L diagram is a scatter plot, as shown also by Bedding \& Zijlstra (1998) for oxygen Miras and by Bergeat et al. (2001) for carbon stars.

2. The Miras and SR are partly separated on this diagram (cf. also Bedding \& Zijlstra 1998). The Miras have longer periods (no star with a period shorter than 120 days is a Mira), but both types of variables have similar luminosity ranges.

3. The separation is not complete. Several SR are located in the Mira region, and vice versa.

4. The pulsation characteristics of carbon and oxygen-rich Miras are indistinguishable.

5. The SR P-L sequence, if there is one, has a different slope from that of the Miras.

6. The partial overlapping of the Mira and SRb regions implies that some Miras may switch to SRb and vice-versa. As we discuss in the next section, there is ample evidence for mode-switching between Mira-like and SR-like pulsation modes, and the incomplete segregation in Fig. 4 may be due to this.
7. The (relatively small) number of SRa stars are spread approximately equally between the Mira and SRb regions of the diagram.

8. Both the Mira and SR variables have a maximum luminosity of $M_{K}=-8.2 \pm 0.2$. The maximum luminosity also holds for both oxygen and carbon stars, and is in agreement with the value found for LMC stars.

\subsection{The period-luminosity relationship}

Many of the objects do not have significant parallaxes, even after reprocessing the Hipparcos data, and absolute magnitudes derived from the subset of objects in Tables 1 and 2, limited to Miras and SRb, and depicted in Fig. 4 which are selected by parallax (and/or by $\varpi / \varepsilon(\varpi)$ ) will be biased (Lutz \& Kelker 1973). While the Hipparcos catalogue is magnitude limited and therefore absolute magnitudes are also subject to Malmquist bias, the parallax for an LPV may become undetectable with respect to stars of similar brightness before the star does - a star with $M_{K}=-8$ at $D=1 \mathrm{kpc}$ has $V \sim 8-$ and errors on the parallaxes are therefore the major source of uncertainty.

Although the astrometry based luminosity (ABL, Arenou \& Luri 1999) is an unbiased estimator of the inverse of the luminosity, it cannot be used directly to calculate absolute magnitudes which can then be used to derive a period-luminosity relation: the symmetry of the error bars on ABL does not propagate to the absolute magnitude. Instead of fitting the absolute magnitudes, we work in parallax space, where the errors are Gaussian. Therefore, instead of fitting Eq. (1), we fit

$\varpi=P^{\frac{a}{5}} 10^{\frac{b}{5}} 10^{\frac{10-K+A_{K}}{5}}$

where $\varpi$, the parallax in mas, can be used regardless of its sign. The parameters are fitted by chi-square minimization using non-linear optimization techniques, and their uncertainties are obtained by Monte Carlo simulations.

Figure 5 shows the resulting mean $M_{K}$ vs. $\log P$ diagram for Miras. The grouping was done by sorting the data by period, and making bins which contained at least two stars with parallaxes measured at the $2 \sigma$ level or better. The mean period was calculated for each bin by simply averaging the periods, while the mean absolute $K$ magnitude was derived from the ABL (Arenou \& Luri 1999). The data in that figure are strongly correlated: the linear correlation coefficient is 0.84 , giving a $\gg 99 \%$ chance of correlation. The least-squares fit to the $\mathrm{P}-\mathrm{L}$ relationship for Miras gives

$M_{K}=-3.39( \pm 0.47) \log P($ days $)+0.95( \pm 3.01)$

which is very consistent with that found by Feast et al. (1989) and (Whitelock \& Feast 2000) as shown in Fig. 5.

Figure 5 also shows P-L data for three nearby OH/IR stars with reliable phase-lag distances (van Langevelde et al. 1990): WX Psc (IRC+10011), RR Aql and R Aql. The last object is nearby enough to be measured by Hipparcos: the phase lag distance, $290 \mathrm{pc}$, is in reasonably good agreement with the Hipparcos parallax $\left(\varpi^{-1}=235 \pm 52 \mathrm{pc}\right)$. These data also show reasonable agreement with the derived $\mathrm{P}-\mathrm{L}$ slope. 


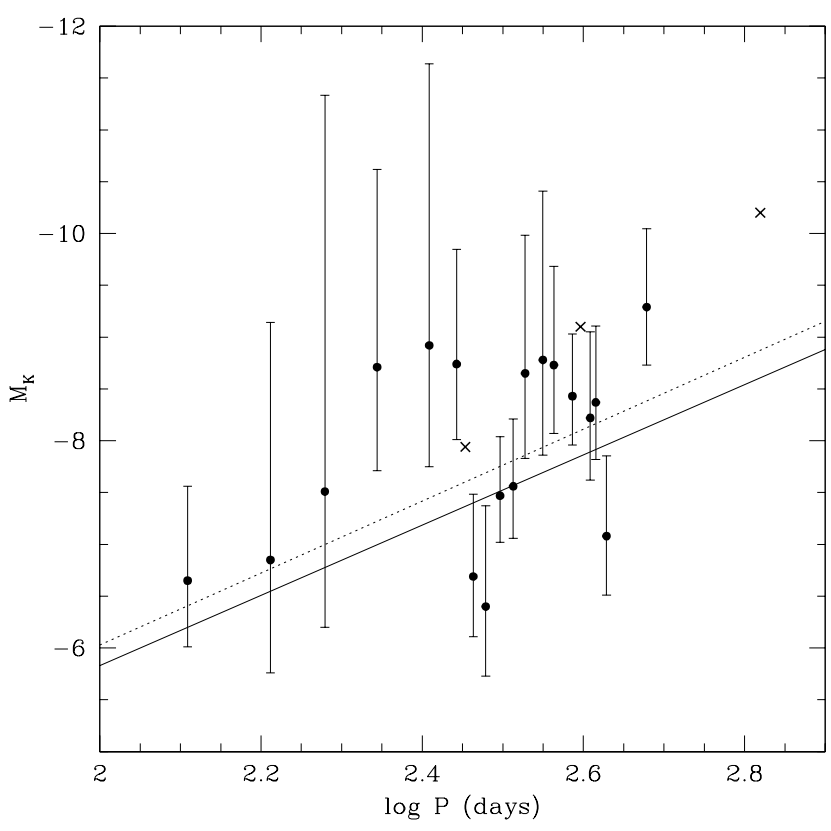

Fig. 5. Binned P-L data for Mira variables. The LMC relationship of Feast et al. (1989) is shown by the dotted line, and the least-squares fit to the data by the solid line. Crosses: oxygen-rich Mira variables with distances from $\mathrm{OH}$ phase-lag measurements (see text).

The data for the SR variables also show a correlation, though less strongly: the correlation coefficient is 0.65 , giving a $>90 \%$ chance of correlation. The least-squares fit is:

$M_{K}=-1.34( \pm 0.06) \log P($ days $)-4.5( \pm 0.35)$.

The above results disagree with those of Barthès et al. (1999) who divide their data into different samples, finding different relations for each sample, with slopes shallower than the LMC Mira slope.

\section{Variability and mass loss}

Miras with periods as long as nearly 2000 days have been found. These are all OH/IR stars with large mass loss rates. As discussed below, mass loss becomes very high for LPVs, completely obscuring the star at visible and near-infrared wavelengths. Thus a large part of the period space occupied by LPVs is inaccessible to studies of the P-L relationship because parallax values for these stars are measured at present only at optical wavelengths. However, there are indications that the $\mathrm{P}-\mathrm{L}$ relationships so far found do not hold for very long period Miras. First, if it did, the predicted bolometric luminosity for a star with a $2000 \mathrm{~d}$ period would be $7 \times 10^{4} L_{\odot}$, well in excess of the maximum AGB luminosity of $5 \times 10^{4} L_{\odot}$ (Paczyński 1970). Recently, He \& Chen (2001) have examined the P-L relationship for OH/IR stars with phase-lag or kinematic distances; there is a large amount of scatter in the $\mathrm{P}-\mathrm{L}$ diagram, and the relationship may be different for objects in different IRAS spectral classes. Furthermore, the bolometric luminosities of many of these highly obscured, very long period stars are relatively modest, often $<10^{4} L_{\odot}$.

The CGCVS contains very few stars with periods longer than 400 days. However, such stars are known, for example the
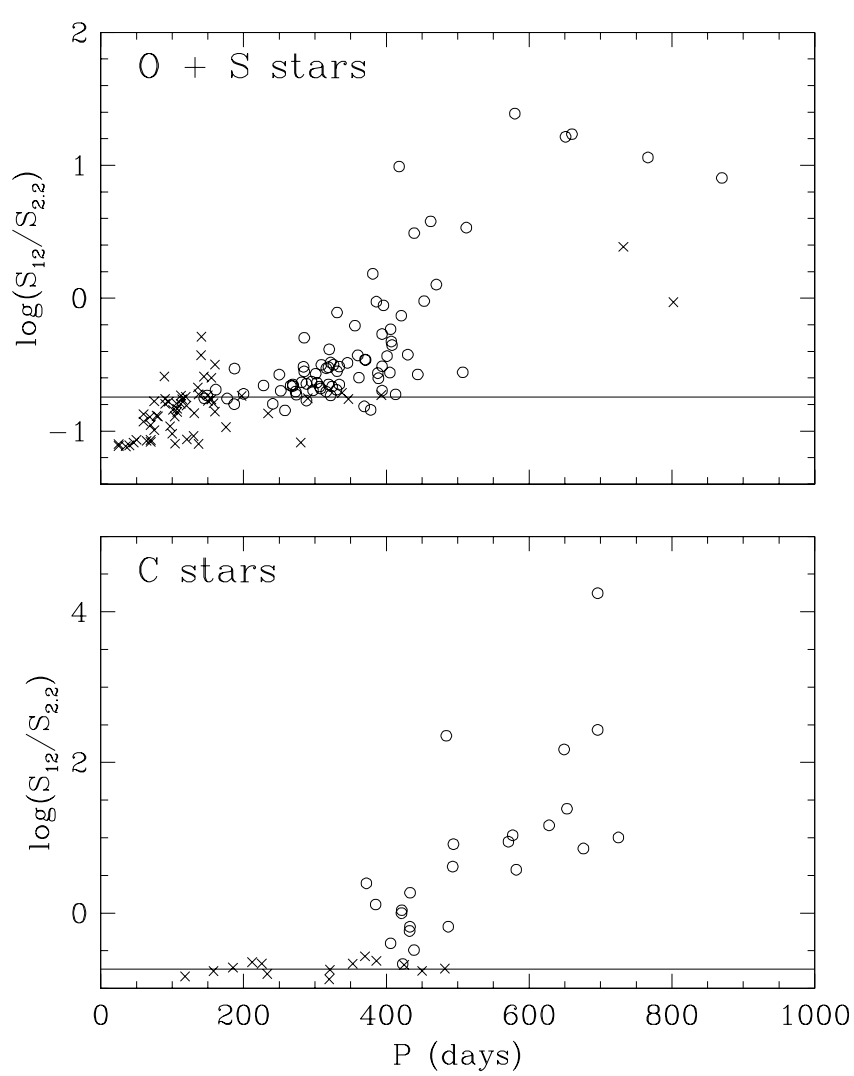

Fig. 6. Infrared color $\left(S_{12} / S_{2.2}\right)$ versus period for oxygen stars (upper panel) and carbon stars (lower panel). Open symbols: Mira variables. Crosses: SR/SRb variables. The horizontal line shows the $S_{12} / S_{2.2}$ for a $2500 \mathrm{~K}$ black body.

$\mathrm{OH} / \mathrm{IR}$ stars. Le Bertre $(1992,1993)$ measured near infrared light curves for several tens of oxygen-rich and carbon stars which were first discovered in infrared sky surveys. These objects are heavily obscured, and almost all of them have periods longer than 400 days. Almost all of them vary with large amplitudes and are therefore Mira variables. In Fig. 6, the IR colors of these stars are compared with those from Tables 1 and 2, showing the period versus the ratio of the $12 \mu \mathrm{m} / 2.2 \mu \mathrm{m}$ flux densities (as noted in Sect. 2, this quantity is a good indicator of mass loss). The objects plotted are those from Tables 1 and 2 for which we have the appropriate measurements. Also plotted are the stars for which Le Bertre $(1992,1993)$ measured periods (with the exception of the OH/IR stars, which are often at large distances and therefore have an unknown amount of interstellar extinction), and, to round out the samples, the carbon star CIT 6 (period from Alksnis 1995) and the oxygen supergiants VY CMa (Mira) and VX Sgr (SRb).

Figure 6 shows that copious mass loss turns on more-or-less abruptly at periods of about 300-350 days for both oxygen and carbon stars. Further, the mass loss rate is much higher for Miras at a given period than it is for SR variables (the flattening in $S_{12} / S_{2.2}$ for oxygen/S stars at long periods is due to the saturation of the $12 \mu \mathrm{m}$ flux as the $9.7 \mu \mathrm{m}$ silicate feature goes into absorption with increasing optical depth). Stars with high mass loss rates are essentially invisible at optical wavelengths. Thus the $\mathrm{P}-\mathrm{L}$ relation is incomplete: stars with periods longer than 


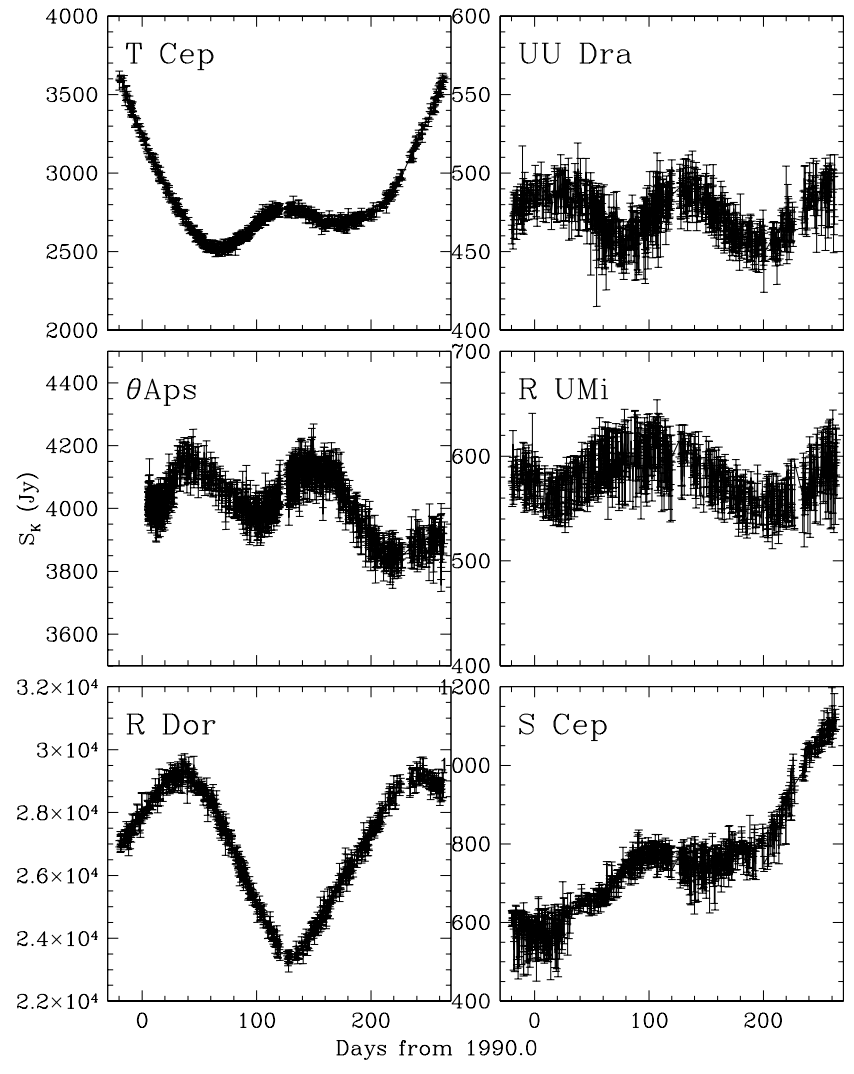

Fig. 7. Example COBE $K$ band light curves for LPVs. T Cep and S Cep are Miras, R UMi is SRa, and the other stars are SR. A small number of data points with large variance have been removed. Both T Cep and S Cep show the bump on the rising part of the light curve often seen for Miras (Lockwood \& Wing 1971).

about 400 days are absent. This part of period space will only become accessible by astrometry at the few microarcseconds level or better at infrared wavelengths.

\section{Variability modes in LPVs}

Figure 7 illustrates some additional problems with studying the $\mathrm{P}-\mathrm{L}$ relation for LPVs. It shows DIRBE $2.2 \mu \mathrm{m}$ light curves for six variable stars for which almost complete time coverage is available: two Miras (S Cep and T Cep), one SRa (R UMi) and three $\mathrm{SRb}(\theta$ Aps, UU Dra and R Dor). The SR variables all have periods short enough that a complete phase information is available, and the $\mathrm{SRa}, \mathrm{R} \mathrm{UMi}$, is indistinguishable in its variability characteristics from the other SR stars. Both Mira variables have periods that are longer than the COBE time coverage of 300 days: T Cep's period is $388 \mathrm{~d}$, S Cep's $488 \mathrm{~d}$. The partial COBE light curves are consistent with this period.

Table 3 lists period data from DIRBE, where available, and from the literature, for stars with multiple periods, all of which are in the sample analyzed earlier in this paper. Kerschbaum et al. (2001) note that fewer than $50 \%$ of the periods for $\mathrm{SRb}$ stars determined from their monitoring observations agree with those from the CGCVS: the data in Table 3 support this conclusion.

Several analyses of very long time series data for individual stars have recently appeared in the literature. Bedding
Table 3. Identified periods of variability.

\begin{tabular}{|c|c|c|c|c|}
\hline HIP & CGCVS & var & $\mathrm{P}$ & Ref. \\
\hline \multirow[t]{3}{*}{21479} & R Dor & $\mathrm{SRb}$ & 209 & DIRBE \\
\hline & & & 175,332 & Bedding \& Zijlstra (1998) \\
\hline & & & 338 & CGCVS \\
\hline \multirow[t]{2}{*}{28166} & BQ Ori & SRa & 127,240 & Kiss et al. (1999) \\
\hline & & & 110 & CGCVS \\
\hline \multirow[t]{3}{*}{36288} & Y Lyn & SRc & 133,205 & Szatmary et al. (1996) \\
\hline & & & 110,1400 & Percy et al. (2001) \\
\hline & & & 110 & CGCVS \\
\hline \multirow[t]{2}{*}{53085} & V Hya & SRa & 530,6000 & Knapp et al. (1999) \\
\hline & & & 529 & CGCVS \\
\hline \multirow[t]{2}{*}{63950} & FS Com & $\mathrm{SRb}$ & 55,600 & Percy et al. (2001) \\
\hline & & & 58 & CGCVS \\
\hline \multirow[t]{2}{*}{65835} & R Hya & M & (314) & DIRBE \\
\hline & & & 388 & CGCVS \\
\hline \multirow[t]{2}{*}{67419} & W Hya & SRa & 274 & DIRBE \\
\hline & & & 382 & CGCVS \\
\hline \multirow[t]{2}{*}{68815} & $\theta$ Aps & $\mathrm{SRb}$ & 121 & DIRBE \\
\hline & & & 119 & CGCVS \\
\hline \multirow[t]{2}{*}{70969} & Y Cen & $\mathrm{SRb}$ & (338) & DIRBE \\
\hline & & & 180 & CGCVS \\
\hline \multirow[t]{3}{*}{80802} & R UMi & SRa & 181 & DIRBE \\
\hline & & & 170,325 & Kiss et al. (1999) \\
\hline & & & 324 & CGCVS \\
\hline \multirow[t]{3}{*}{81747} & AX Sco & SR & (39), (64) & Kerschbaum et al. (2001) \\
\hline & & & 124,128 & Kerschbaum et al. (2001) \\
\hline & & & 138 & CGCVS \\
\hline \multirow[t]{2}{*}{81835} & S Dra & $\mathrm{SRb}$ & 182 & DIRBE \\
\hline & & & 136 & CGCVS \\
\hline \multirow[t]{3}{*}{95173} & T Sge & SR & (112) & Kerschbaum et al. (2001) \\
\hline & & & 178,316 & Kerschbaum et al. (2001) \\
\hline & & & 166 & CGCVS \\
\hline \multirow[t]{2}{*}{100605} & UU Dra & $\mathrm{SRb}$ & 117 & DIRBE \\
\hline & & & 120 & CGCVS \\
\hline \multirow[t]{2}{*}{104451} & T Cep & M & $112,>290$ & DIRBE \\
\hline & & & 388 & CGCVS \\
\hline
\end{tabular}

et al. (1998) find two periods, $175 \mathrm{~d}$ and $332 \mathrm{~d}$, for R Dor, with switching between the two periods on a timescale of about 1000 days. The DIRBE light curve has an intermediate period of $209 \mathrm{~d}$, intermediate between the long and short periods. Bedding et al. (1998) note that the longer period, plus the star's well-determined absolute magnitude, place it on the LMC Mira P-L relation (note that it also lies on the Mira $\mathrm{P}-\mathrm{L}$ relation found above) and suggest that the star switches pulsation modes between SR and Mira variability, i.e. between third and first overtone radial pulsation, with Miras considered to be pulsating in the first overtone (Wood 1990; Willson 2000) - although see the discussion by Barthès (1998).

As pointed out by Lebzelter \& Kiss (2001) understanding LPVs requires both short- and long-term monitoring. Other studies of long time-series for LPVs include that of Howarth $\&$ Greaves (2001) who find two periods for the Mira variable $\mathrm{T}$ Cas, $445 \mathrm{~d}$ and $222 \mathrm{~d}$, whose ratio suggests that the longer period is the fundamental (cf. Barthès 1998); switching between 
these two modes takes place on a time scale of about $3000 \mathrm{~d}$. Szatmary et al. (1996) and Bedding et al. (1998) find that V Boo appears to have changed from a Mira to an SR variable. Cadmus et al. (1991) also find evidence of mode switching for several stars. Sterken et al. (1999) show that the period of $\chi$ Cyg is increasing. One star which does not appear to undergo such behavior is V Hya, for which Knapp et al. (1999) find repeatable and well established periods of $530 \mathrm{~d}$ and $6000 \mathrm{~d}$. Finally, Marengo et al. (2001) examine the structure of circumstellar shells due to mass loss, in which the time-history of that mass loss may be seen, and suggest that essentially all LPVs undergo mode switching between the Mira and semi-regular phases.

\section{Discussion and conclusions}

In this paper, we have examined the distribution of LPVs in nearby regions of the Galaxy in the $P-M_{K}$ plane using distances derived from Hipparcos parallaxes. We find P-L relationships for both Mira and SRb variables. The slope for the $\mathrm{SRb}$ is much shallower than that for the Miras. The relationships found herein do not agree with some previous relationships found in the literature. The $\mathrm{P}-\mathrm{L}$ relationship for Miras agrees well with that found in the LMC. We discuss the results from the present paper and from the literature on period changes in LPVs and on mass loss and its dependence on the variability period. The evidence suggests that LPVs, at least those with periods $<400 \mathrm{~d}$, may change their variability modes back and forth between Mira and SR variation, with models suggesting that different radial pulsation modes become dominant. However, the P-L relationships found here do not extend to periods longer than about 700 days.

If visible LPVs, i.e. those with low mass loss rates, switch between the Mira and SR modes, the shallowness of the P-L relationship for $\mathrm{SRb}$ relative to that for Miras is easy to understand: the period of a given star may decrease during the Mira $\rightarrow$ SRb phase by a factor of 2-4 (depending on the pulsation modes) but the luminosity stays about the same.

We also show that mass loss is strongly dependent on period and variability mode. Miras at a given period are losing much more mass than are SR, and the mass loss rate rises steadily with period for both Miras and SRb. Stars with periods longer than about 400-500 days are losing so much mass that they become highly obscured at visible wavelengths and have no parallax information: thus a large part of period space is not available for studies of the $\mathrm{P}-\mathrm{L}$ relations. These very long period variables are almost all Miras, suggesting that the mode switching characteristic of shorter-period red variables disappears as the period lengthens.

Comparing the P-L relationships for LPVs from the literature and this paper, it appears that the results are strongly dependent on the sample selection. The use of $\mathrm{P}-\mathrm{L}$ relationships to determine distances to LPVs is therefore not likely to be useful in at least the near future. However, the maximum $K$ absolute magnitude in the nearby (few hundred parsecs) region of the Galaxy is $-8.2 \pm 0.2$, in good agreement with observations of the LMC. This corresponds to a bolometric luminosity of about $5000-6000 L_{\odot}$, about the maximum possible luminosity for AGB stars. This result, in itself, shows the unlikeliness of a $\mathrm{P}-\mathrm{L}$ relationship which extends to periods longer than 400-500 days: stars with shorter periods are already close to the AGB luminosity limit.

Further progress on studying the $\mathrm{P}-\mathrm{L}$ characteristics of LPVs would be greatly aided by astrometric measurements at the few microarcsecond level in near-infrared bands, which would make possible the analysis of the entire period range.

Acknowledgements. We thank the referee, F. van Leeuwen, and M. Feast for many valuable comments. We are very grateful to NASA for generous support of this work via grant NAG5-11094 as well as to ESA via PRODEX C15152/01/NL/SFe(IC). This research made use of the SIMBAD database, operated at CDS, Strasbourg, France; of the NASA Astrophysics Data System, operated at the Harvard-Smithsonian Center for Astrophysics; of the astro-ph electronic preprint server operated by Cornell University; and of the data analysis and display program SM, written by Robert Lupton and Patricia Monger. We used archival data from the Hipparcos mission, operated by the European Space Agency, and from the COBE mission, operated by NASA via the Goddard Space Flight Center.

\section{References}

Alard, C., Blommaert, J. A. D. L., Cesarsky, C., et al. 2001, ApJ, 552, 289

Alksnis, A. 1995, Baltic Astron., 4, 79

Alvarez, R., Jorissen, A., Plez, B., et al. 2001, A\&A, 379, 305

Alvarez, R., Mennessier, M. O., Barthès, D., Luri, X., \& Mattei, J. A. 1997, A\&A, 327, 656

Arenou, F., \& Luri, X. 1999, in Harmonizing Cosmic Distance Scales in a Post-Hipparcos Era, ed. D. Egret, \& A. Heck, ASP Conf. Ser., 167,13

Barthès, D., Luri, X., Alvarez, R., \& Mennessier, M. O. 1999, A\&AS, 140,55

Barthès, D. 1998, A\&A, 333, 647

Bedding, T. R., \& Zijlstra, A. A. 1998, ApJ, 506, L47

Bedding, T. R., Zijlstra, A. A., Jones, A., \& Foster, G. 1998, MNRAS, 301, 1073

Beichman, C. A., Neugebauer, G., Habing, H. J., Clegg, E., \& Chester, T. J. 1988, IRAS Catalogue and Atlases: Explanatory Supplement (GPO)

Bergeat, J., Knapik, A., \& Rutily, B. 2001, A\&A, 369, 178

Bessell, M. S., \& Brett, J. M. 1988, PASP, 100, 1131

Boggess, N. W., Mather, J. C., Weiss, R., et al. 1992, ApJ, 397, 420

Cadmus, R. R., Willson, L. A., Sneden, C., \& Mattei, J. A. 1991, AJ, 101,1043

Cardelli, J. A., Clayton, G. C., \& Mathis, J. S. 1989, ApJ, 345, 245

Cioni, M. R. L., Marquette, J. B., Loup, C., et al. 2001, A\&A, 377, 945

ESA 1997, The Hipparcos and Tycho Catalogues (ESA SP-1200)

Feast, M. W., Glass, I. S., Whitelock, P. A., \& Catchpole, R. M. 1989, MNRAS, 241, 375

Gezari, D. Y., Pitts, P. S., \& Schmitz, M. 1999, Catalog of Infrared Observations, 5th edn.

Glass, I. S., \& Lloyd Evans, T. 1981, Nature, 291, 303

Groenewegen, M. A. T., \& Whitelock, P. A. 1996, MNRAS, 281, 1347

Hakkila, J., Myers, J. M., Stidham, B. J., \& Hartmann, D. H. 1997, AJ, 114, 2042

Hauser, M. G., Kelsall, T., Leisawitz, D., \& Weiland, J. 1998, COBE Diffuse Infrared Background Experiment (DIRBE) Explanatory Supplement, Vol. 2.3

He, J. H., \& Chen, P. S. 2001, AJ, 121, 2752 
Howarth, J. J., \& Greaves, J. 2001, MNRAS, 325, 1383

Ivezić, Ž., \& Elitzur, M. 1997, MNRAS, 287, 799

Jorissen, A., \& Knapp, G. R. 1998, A\&AS, 129, 363

Jura, M., \& Kleinmann, S. G. 1992, ApJS, 79, 105

Kerschbaum, F., \& Hron, J. 1994, A\&AS, 106, 397

Kerschbaum, F., Lebzelter, T., \& Lazaro, C. 2001, A\&A, 375, 527

Kholopov, P. N., Samus, N. N., Frolov, M. S., et al. 1998, Combined General Catalogue of Variable Stars, 4th edn.

Kiss, L. L., Szatmáry, K., Cadmus, Jr., R. R., \& Mattei, J. A. 1999, A\&A, 346, 552

Knapp, G., Pourbaix, D., \& Jorissen, A. 2001, A\&A, 371, 222

Knapp, G. R., Dobrovolsky, S. I., Ivezić, Ž., et al. 1999, A\&A, 351, 97

Knapp, G. R., Sandell, G., \& Robson, E. J. 1993, ApJS, 88, 173

Le Bertre, T. 1992, A\&AS, 94, 377

Le Bertre, T. 1993, A\&AS, 97, 729

Lebzelter, T., \& Kiss, L. L. 2001, A\&A, 380, 388

Lockwood, C. W., \& Wing, R. F. 1971, ApJ, 169, 63

Lutz, T. E., \& Kelker, D. H. 1973, PASP, 85, 173

Marengo, M., Ivezić, Ž., \& Knapp, G. R. 2001, MNRAS, 324, 1117

Neugebauer, G., \& Leighton, R. B. 1969, Two-Micro Sky Survey Catalogue (NASA SP-3047)
Paczyński, B. 1970, Acta Astronomica, 20, 47

Percy, J. R., Wilson, J. B., \& Henry, G. W. 2001, PASP, 113, 983

Platais, I., Pourbaix, D., Jorissen, A., et al. 2003, A\&A, 397, 997

Pourbaix, D., Platais, I., Detournay, S., et al. 2003, A\&A, 399, 1167

Reid, M. J., \& Goldston, J. E. 2002, ApJ, 568, 913

Smith, B. J., Leisawitz, D., Castelaz, M. W., \& Luttermoser, D. 2002, AJ, 123, 948

Sterken, C., Broens, E., \& Koen, C. 1999, A\&A, 342, 167

Szatmary, K., Gal, J., \& Kiss, L. L. 1996, A\&A, 308, 791

van Langevelde, H. J., van der Heiden, R., \& van Schooneveld, C. 1990, A\&A, 239, 193

van Leeuwen, F., Feast, M. W., Whitelock, P. A., \& Yudin, B. 1997, MNRAS, 287, 955

Whitelock, P., \& Feast, M. 2000, MNRAS, 319, 759

Whitelock, P., Marang, F., \& Feast, M. 2000, MNRAS, 319, 728

Willson, L. A. 2000, ARA\&A, 38, 573

Wood, P. R. 1990, in From Miras to Planetary Nebulae: Which path for stellar evolution?, ed. M. O. Mennessier, \& A. Omont (Éditions Frontières), 67

Wood, P. R. 2000, PASA, 17, 18

Zijlstra, A. A., Bedding, T. R., \& Mattei, J. A. 2002, MNRAS, 334, 498 